\title{
First report of Diplodia corticola in Greece on kermes oak (Quercus coccifera)
}

\author{
P. Tsopelas ${ }^{1}$, B. Slippers ${ }^{2}$, Z. Gonou-Zagou ${ }^{3}$, M. J. Wingfield ${ }^{2}$
}

(1) NAGREF-Institute of Mediterranean Forest Ecosystems, Terma Alkmanos, 11528 Athens, Greece

(2) Department of Genetics Forestry and Agricultural Biotechnology Institute, University of Pretoria, Pretoria, South Africa

(3) Faculty of Biology, University of Athens, Panepistimioupolis, 15784 Athens, Greece

\section{P. Tsopelas \\ Email: tsop@fria.gr}

In 2007, symptoms of extensive branch and shoot dieback were observed on kermes oak (Quercus coccifera) shrubs and trees in some localities in the Messinia prefecture of south western Peloponnese, Greece. Symptoms were more intense during summer, with abundant dead branches and twigs having wilted leaves. On close examination, cankers were detected on the branches and twigs showing symptoms. Cankers were also evident on larger branches with no apparent foliar symptoms. In many of the branch samples taken, dark brown to black pycnidia were observed emerging through the bark on the canker surfaces.

Fungal isolates from bark and wood tissues on symptom-bearing branches, as well as from spore masses in the pycnidia, were morphologically very similar and typical of species of the Botryosphaeriaceae. Colonies on malt extract agar (MEA) showed dense aerial mycelium that was initially white, gradually becoming dark grey olivaceous, with a radial growth rate of $1.3 \mathrm{~cm} \mathrm{day}^{-1}$ at $25^{\circ} \mathrm{C}$. Conidiomata were formed on sterile poplar twigs placed on cultures grown on water agar and incubated under ultraviolet lights for three to 4 weeks. Conidia formed in culture and those from pycnidia on cankers were similar in shape and size: cylindrical with rounded ends, hyaline and unicellular, becoming light brown and two-celled with age, (20-)24-32(-38) $\times(10-) 12-16(-17) \mu \mathrm{m}$. Morphological characteristics and nucleotide sequences of the ITS region of ribosomal DNA (GenBank Accession nos. GQ396149-GQ396153) confirmed the identification of the fungus as Diplodia corticola (teleomorph: Botryosphaeria corticola) described by Alves et al. (2004).

Pathogenicity tests were carried out in the summer of 2008 on Q. coccifera shrubs and trees growing in the same area as the naturally infected plants. Branches $(0.7-5.0 \mathrm{~cm}$ in diameter) were wound inoculated with mycelium on agar plugs. Necrosis of bark and wood tissues was evident on the branches 6 weeks after inoculation, with the formation of characteristic cankers and wood discolouration beneath the cankered area. Pycnidia formed on the majority of inoculated branches. The fungus was consistently re-isolated from necrotic bark and wood tissue, as well as from pycnidial spore masses on the inoculated branches. No symptoms developed on the control branches inoculated with sterile MEA. Diplodia corticola is known to occur on oak species in Spain, Portugal, Italy and Morocco (Alves et al., 2004). This is the first record of D. corticola in Greece and the first host association with $Q$. coccifera in Europe.

\section{Reference}

Alves A, Correia A, Luque J, Phillips A, 2004. Botryosphaeria corticola, sp. nov. on Quercus species, with notes and description of Botryosphaeria stevensii and its anamorph, Diplodia mutila. Mycologia 96, 598-613. 